\title{
Active and Reactive Powr Control of Dcig Wind Power System using Evolutionary Algorithm Based Fraction Order Controllers.
}

\author{
M.Vasavi Uma Maheswari, P.V.Ramana Rao
}

\begin{abstract}
This paper commences an exalted control scenario for Wind Energy Systems(WES) adopting Doubly Cater Induction Generator (DCIG) . A vigorous Ant Lion Optimizer(ALO) technique is assented with a Fractional Order PI assessor to optimize the powers and to lift the aggressive performance of WES[2][3]. The enforcement and adequacy of ALOFOPI assessor shows amusing countenance in terms of blather devaluation confined concurrence time and hefty against specifications[1]. The proposed ALOFOPI algorithm shows a great convergence and enhanced stability.
\end{abstract}

Keywords: Wind Energy Systems(WES),Doubly Cater Induction Generator(DCIG), Ant Lion Optimizer(ALO), Fractional Order PI assessor(FOPI), PI assessor.

\section{INTRODUCTION}

As Wind Energy(WE) is a continual reserve with no ammunition charge and no consumptive debate gases and are originated consequently[3]. WE is the backup for exploring and evolution of power generation. The wind is an innate development against many causes allying clime disparities, barometrical pressures and the earth radiation fogs. The above mentioned aspects invent the wind acceleration and potential for electrical power generation. WE is reformed into electrical energy by employing Wind Turbine(WT) they novitiate driving force to electromotive force. In this we study about the control entities in DCIG,constates the main stream contours for the WT in the exploring exertions.

This paper is reorganized as proceeds in part I depicts about the WES [3]firmness to the yield potential from wind, the power coefficient $\left(\mathrm{C}_{0}\right)$ and the Tip Speed Ratio Characteristic[3]. DCIG exemplary and the curb strategy is accustomed by a PI assessor in part II [3] and mutated PI assessors in part III and IV respectively[4]. And part V is counterfeit results with matlab Simulink model and final cessation in part VI.

PartI Wind Energy System(WES):

Revised Manuscript Received on April 12, 2019.

M.Vasavi Uma Maheswari,Asst Professor EEE DepartmentCollege of Engineering \& Technology AcharyaNagarjuna University Guntur,Andhra Pradesh.T.N, (vasavimajety.eee@gmail.com)

P.V.RamanaRao,Professor EEE Department College of Engineering \& Technology AcharyaNagarjuna University Guntur, Andhra PradeshT.N, India. (pvrr.eee@gmail.com)

S.V. Jayaram Kumar,Professor EEE Department College of Engineering, JNTUHHyderabad. Telangana, T.N, India. (Svjkumar101@rediffmail.com)

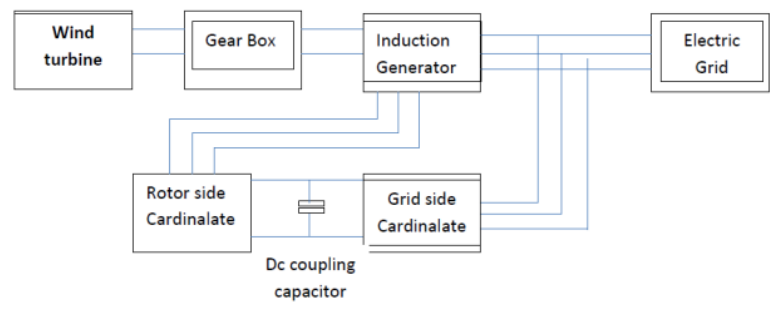

Fig1:Block diagram of DCIG system

WES will metamorphose the dynamic vitality into automa ted vitality by WT blades and yet novitiates to the electrical vitality through a dynamo. The WT based on DCIG scheme is given in fig1. The vitality originated form the WT's depends on the velocity of wind speeds. At flat wind speeds the WT cannote provoke electrical vitality i.e for $(1-3) \mathrm{m} / \mathrm{s}$. At wind speeds in the midst $(2-5) \mathrm{m} / \mathrm{s}$ the WT's will start calling i.e 'Cut-in-wind-speed'. At the wind speeds in the midst of $(12-15) \mathrm{m} / \mathrm{s}$ is termed as the "nominal or rated wind speed', where WT's employing on their full spectrum. At huge wind speeds that are over $25 \mathrm{~m} / \mathrm{s}$, the WT will be hampered i.e , because huge wind speeds may deteriorate the mechanics of the WT's. The gain of WT is dependent on the power coefficient $\mathrm{C}_{\mathrm{o}}$. It is given by

$$
P \mathrm{~m}=\frac{1}{2} \mathrm{p} \pi \boldsymbol{Y}^{2} \mathrm{y}^{3} C_{\mathrm{o}}------(\mathbf{1})
$$

And the tip speed ratio is constructed as

$$
\Gamma=\frac{\mathrm{W}_{\mathrm{r}} \gamma}{\mathrm{y}}-------(
$$

P- is the air density $(\mathrm{kg} / \mathrm{m} 3)$, $\mathrm{y}$ - is velocity of wind speed $\mathrm{m} / \mathrm{s}, W_{\mathrm{r}}-$ is the turbine speed, Co-power coefficient and $\dot{\mathrm{B}}-$ is the pitch angle.

$$
C \mathrm{O}(\Gamma, \dot{\mathrm{B}})=c 1\left(\frac{c 2}{\Gamma i}-C 3 \dot{\mathrm{B}}-C 4\right) e^{\frac{c 5}{\Gamma i}}+C 6--(3)
$$


In which

$$
\frac{1}{\Gamma i}=\frac{1}{\Gamma+0.08 \dot{\mathrm{B}}}-\frac{0.035}{\dot{\mathrm{B}}^{3}+1}
$$

The above parameters depends on the shape of the blade and its aerodynamic consummation. Fig2 shows the affinity among (Co and $\Gamma)$

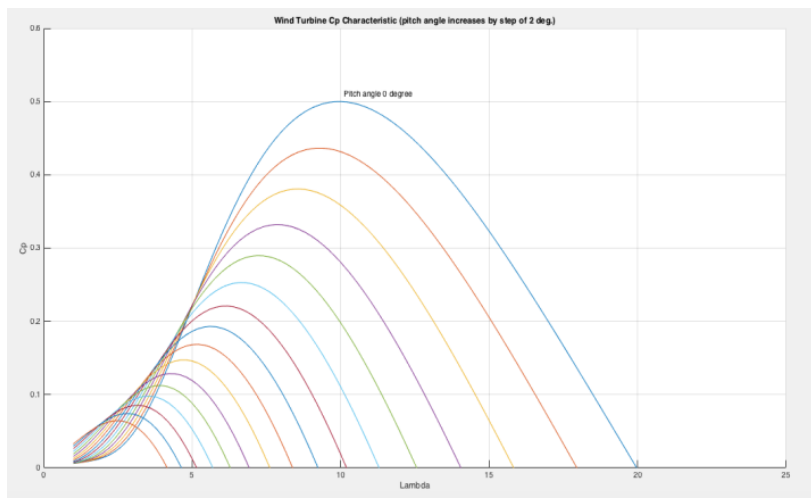

Fig2: plot between Lambda and Power coefficient

\section{Part II DCIG system:}

DCIG consist of WRIG ( wound rotor Induction Generator) and an AC/DC/AC IGBT-placed PWM converter. All electrical valuables and criterions and accredit to the stator as show below in fig3

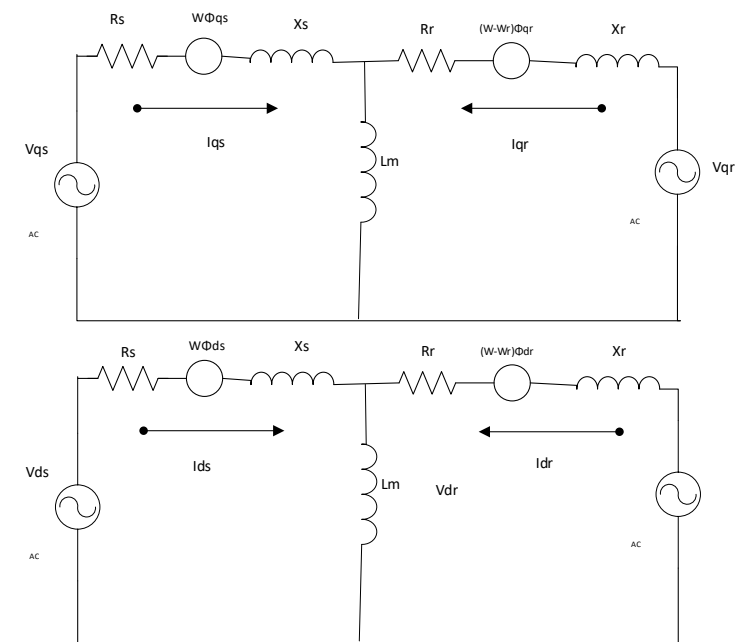

Fig3\&4: proportionate equivalent circuit of DCIG Qaxis and D-axis parameters

The electrical equation of DCIG in the proportionate circuit shown in fig3 and fig4 from the Park's Transformation are given below[2][3]

$$
\begin{gathered}
V d s=R s I d s+\frac{d}{d t} \emptyset d s-W s \emptyset q s-----(5) \\
V q s=R s I q s+\frac{d}{d t} \emptyset d r+W s \emptyset d s----(6) \\
V d r=R r+\frac{d}{d t} \emptyset d r-(W s-W) \emptyset q r--(7) \\
V q r=R r+\frac{d}{d t} \emptyset q r+(W s-W) \emptyset d r---(8)
\end{gathered}
$$

Likewise the stator flux can be conveyed as

$$
\begin{gathered}
\emptyset d r=L s I d s+L m I d r-----(9) \\
\emptyset q r=L r I q r+L m I q s------(10)
\end{gathered}
$$

Where RsRrLsLr acts as resistances and leakage reactance's of both stator and rotor windings and Lm acts as mutual inductance and $\mathrm{W}$-is the rotor speed.

Likewise Vds ,Vdr, Vqs, Vq,r Ids, Idr, Iqs ,Idr, $\phi \mathrm{ds}$, $\phi \mathrm{dr}$, $\phi q s, \phi q r$ acts as the direct and quadrature peripherals of the space phasors of the stator and rotor voltage, current and flux ingredients.

The active and reactive competencies at the stator and rotor are defined as

$P s=V d s I d s+V q s I q s----------(11)$
$Q s=V q s I d s-V d s I q s----------(12)$
$P r=V d r I d r+V q r I q r----------(13)$
$Q r=V q r I d r-V d r I q r----------(14)$

Eventually, the electromagnetic revolution is given as

$$
\begin{gathered}
\text { Te }=1.5 p(\emptyset d s \text { Iqs }-\emptyset q s I d s)------(15) \\
\text { where }(p-\text { polepairs })
\end{gathered}
$$

\section{CONTROL STRATAGEM\& RESULTS:}

An exemplary design of a PI Assessor system is shown in fig 4 below from which the PI assessor is adopted to achieve the proportional and integral behavior of the resulting signals admixed and include to form the control signal $u(t)$ enforced with the plant model. A mathematical depiction of the PI assessor is

$$
\begin{aligned}
& u(t)=\left(K p\left[e(t)+\frac{1}{T i} \int \llbracket e(t) d T\right) U p(t)+U i(t)(16)\right. \\
& \text { where Kp-Propotional gain, Ti } \\
& \text { - integral time constant of PI assessor, } e(t) \\
& - \text { error signa, } u(t)-\text { input signal }=r(t)-y(t)
\end{aligned}
$$

As PI assessors are most recurrently used in which an assessor without Differentiator(D) mode is used during (i) rapid feedback is not enforced (ii) Huge dis orders and turbulences are begun during action of the mean process of assessors(iii) There is only one vitality storage in means (iv) there are huge transit bind in the structure or arrangement.

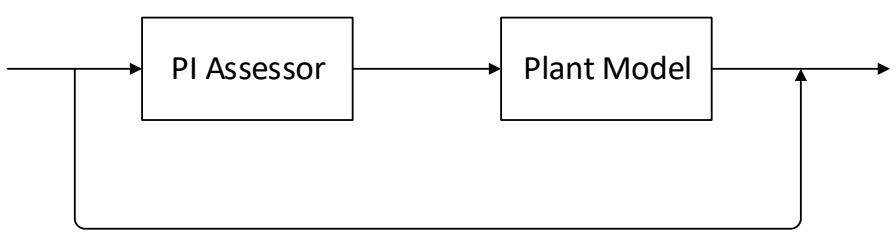

Fig5: an exemplary design of PI control structure

Published By: 


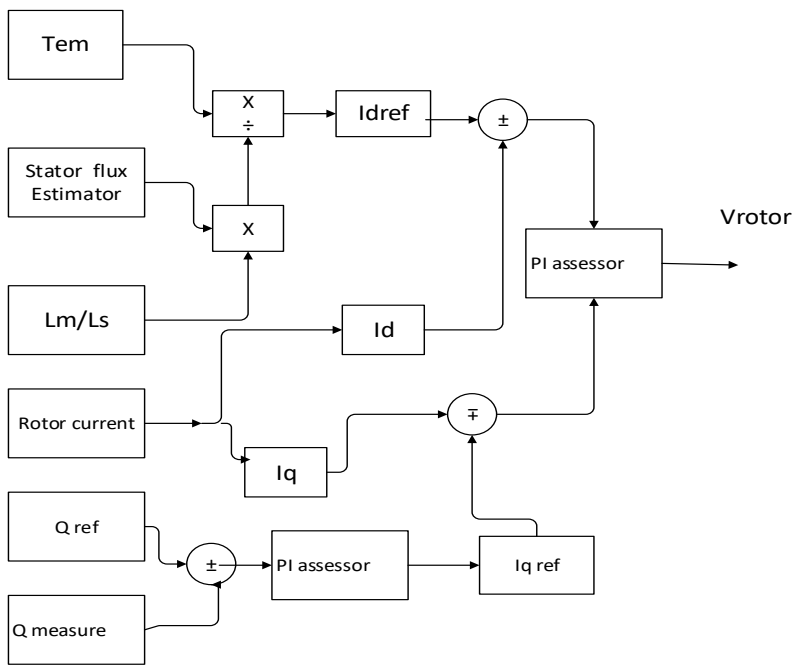

Fig 6: Arrangement of RSC Control System

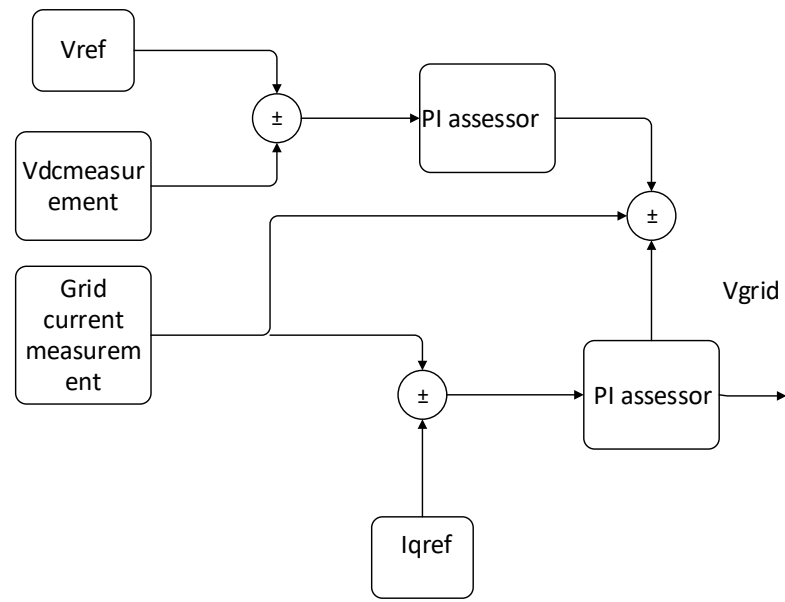

Fig 7: Arrangement of GSC control system

The above arrangements are with a typical PI assessor in which the typical PI assessor is replaced with FOPI assessorand ALOFOPI assessors.

\section{FOPI Assessor:}

From view of typical PI assessor an FOPI assessor abides much sophisticated attainment. It was popularized by Podlubny, who prospected a generalized $\mathrm{PI}^{\lambda} \mathrm{D}^{\mu}$. Adopting a ' $\lambda$ ' order integrator and ' $\mu$ 'order differentiator. He proven the excellence of the FOPID controllers than the typical PID controllers which have three tuning parameters[6]. The generic form of the translation action of the plant was

$$
P(s)=\frac{k}{T s+1}------
$$

where $\mathrm{T}$ and $\mathrm{K}$ are constants. In this sector, two distinct assessors are discussed as ensures:

$$
\begin{gathered}
C p i(s)=K p\left(1+\frac{K i}{s}\right)----(17) \\
\text { Cfopi }=K p\left(1+\frac{K i}{s \lambda}\right)-----(18)
\end{gathered}
$$

\section{Design Stipulation:}

A tune method for PI assessorand FOPIassessor[6] is proposed. We adopt the gain crossover frequency, Wc and phase margin $\phi \mathrm{m}$ to be co equal for the pair of assessors. For the system cohesion and vitality the following restraints are considered.

a) $\operatorname{Arg}[g(j w)]=\operatorname{Arg}[C(j w c) P(j w c)]=\angle C(j w c)+$ $\angle P(j w c)=-\pi+\emptyset m$ Where $\mathrm{G}(\mathrm{jw})$ is the open loop translation action of the system,c(jw) is the assessor translation action and $\mathrm{P}(\mathrm{jw})$ is the plant translation action.

b) Gain cross over frequency restraint:

$$
\mathbf{I} G(j w) I d b=I C(j w c) P(j w c) I d b=0----(19)
$$

c)Vitality to loop yield variations which appeals that the Bode plot to be oblate at the Gain Cross over frequency Wc i.e. the derivative of the open loop phase at the gain cross over frequency to be level to zero

$$
\frac{d(\operatorname{Arg}[G(j w c)]}{d w} \mid=0 \text { at } w=w c------(20)
$$

\section{Fractional order $\mathrm{PI}$ assessor adopting:}

A tune methodfor FOPI assessor is given below for the considered first order plant. The open loop translation action with FOPI assessor is[6]

$$
\begin{aligned}
G(s)=\text { Cfopi } & (s) P(s) \\
& =K p\left(1+\frac{K i}{(j w) \lambda}\right)\left(\frac{K}{T s}+1\right) \\
& --(21)
\end{aligned}
$$

Where $\mathrm{K}$ and $\mathrm{T}$ are known and $\mathrm{Kp}, \mathrm{Ki}$ and $\lambda$ should be designed in the assessor design process. The FOPI assessor can be expressed as

$\operatorname{Cfopi}(s)=K p\left(1+\frac{K i}{s \lambda}\right)=K p\left(1+\frac{K i}{(j w) \lambda}\right)---$

\section{Modelling of ALOPI and ALOFOPI assessors:}

In this annex the ALOPI and ALOFOPI assessors are proposed[1][4]. The approach of tuning PI and FOPIwith ALO is summarized as below. ALO is a recentadvancedstemmer, whichmimes the trapping action of ant lions invariant. There in advent, ants and ant lions act as probeare prospected to find explanation by stride of angling the victim, that comprises the stochastic process of ants,to morgue traps, demurrer of ants in to traps, trappingvictim and reconstruction oftraps. The numerical hypothesisof ALO is depictedas below. Ants step actuarially invariantwhen probingthe victim, so a stochastic of an ant at each stride of accession process is as follows: 
$X i$

$$
\begin{aligned}
& =[0 ; r(1) ; r(1) \\
& +r(2) ; \ldots \ldots \ldots \ldots \ldots ; \sum_{i=1}^{T-1} r(j) ; \sum_{j=1}^{T} r(j)
\end{aligned}
$$

Where $\mathrm{i}=1, \ldots \ldots$, dim, dim is the ant or ant lion tenuity, $\mathrm{T}$ is the maximal number of loops ,

$X=[X 1 ; \ldots \ldots \ldots \ldots$ Xdim $], X i i s a(T+1) \times 1$ matrix and $\mathrm{r}(\mathrm{j})$ is an actuarial expansionand can be utteredas $r=$ 1 rand $>0.5$ orelse -1 forrand $\leq 0.5$ where rand is a ergodic number beget with allocationdiscretionalin the range of $(0,1)$. Discretionalwalks of ants need to be regenerates the location in actual search space according to curtailer and loftier bourne. It is diagnoized by

$$
Y i=\frac{(X i-a i)}{(b i-a i)} \times(d i-c i)+c i-------(24
$$

ai and bi are the littlest and mostlest of $\mathrm{Xi}, \mathrm{Ci}$ and di augur the littlest and mostlest of antlion in the ithtenuity severally respectively, $Y=[Y 1 ; \ldots \ldots \ldots \ldots . . . . \mathrm{dim}]$, $Y i$ is a $(T+1) \times 1$ matrix, $\mathrm{Xi}$ is altered in the domain $[0,1]$ using $\frac{(X i-a i)}{(b i-a i)}$. Then it is regenerated in the sphere [Ci di] using eq(24). It dintthe anomaly aboutthe electant lion[1]. The antsincline are affected by antlions traps. This can be described as

$$
\begin{aligned}
& C=C !+\text { Antlion } \\
& d=d !+\text { Antlion }
\end{aligned}
$$

$\mathrm{c}$ ! and $\mathrm{d}$ ! are the littlest and moistest of dynamic limits at current loop. Ant lion representthe rightant lion electby roulette, according to the fitness[1].

$$
C !=(l b) \div\left(10^{\omega} \times\left(\frac{t}{T !}\right)\right)=(u b) \div\left(10 \omega \times\left(\frac{t}{T !}\right)\right)
$$

' $\mathrm{t}$ ' is the current iteration and lb\&ub are the lower and upper limits of the roulette. Andw-constant based on the current iteration.

$$
\begin{aligned}
& w=2 t>0.1 T \\
& w=3 t>0.5 T \\
& w=4 t>0.75 T \\
& w=5 t>0.9 T \\
& w=6 t>0.95 T
\end{aligned}
$$

$\mathrm{Y}$ is a $(\mathrm{T}+1) \mathrm{Xdim}$ matrix deliberatedin the order of equation

The next step is to adopt elitism to the optimization. Where the pointof each ant depends on the stochasticaround an ant lion selected by the roulette and elite.

$$
\text { Ant }=\frac{R a+R e}{2} \text { Ant is the new position }
$$

$\mathrm{Ra}$ is the random walk around the ant lion selected by the roulette wheel, $\mathrm{Re}$ is the random walk around the Elite.

$$
\text { Antlion }=\text { ant, if } \boldsymbol{f}(\text { Ant })<f(\text { Ant lion }
$$

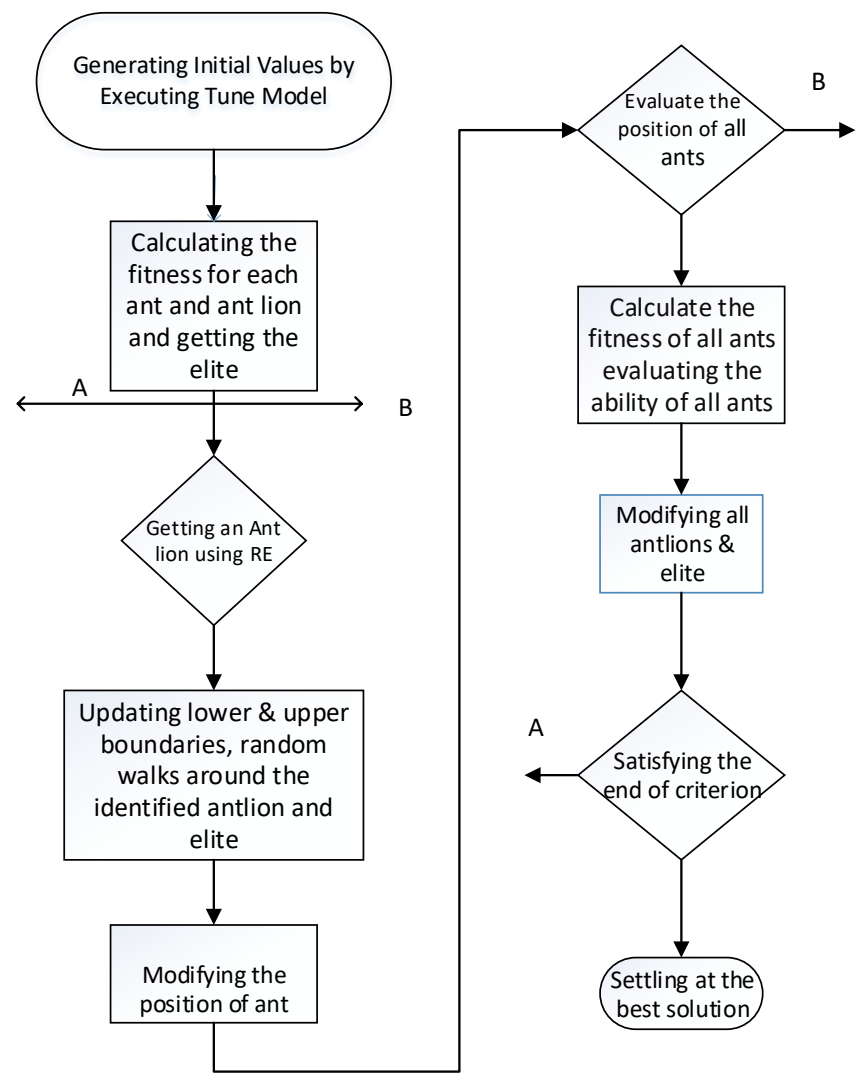

Fig 8: Flow chart for implementing the ALO stemmingrule

\section{Part V Counterfeit Results:}

The case illustrated a wind farm of rating $1.5 \mathrm{MW}$ coupled to DCIG of rating 1.5 $\mathrm{MW}$ is connected to a grid connected system of $30 \mathrm{~km}$ length. This system is estimated the performance with some assumed power setting corresponding to the output active and reactive output powers. The control systems uses a Torque control to stabilize the speed with $1.2 \mathrm{pu}$. To have constant power at the grid it is required to maintain the DC voltage between the back to back converters is maintained constant. To have constant air gap flux (i.e. V/F) ratio is maintained constant such that the air gap power and hence the power from the stator feeding the grid can also be maintained with constant value. At $\dot{B}=0$ degrees the power coefficient is equal to 0.47 . The simulation block diagram is given as below. Lastly the results are analyzed by considering a voltage dip from 0.03 to 0.13 secs with a voltage dip of 50 percent. Following figures shows the output waveforms for a typical PI assessor, PI assessor tuned with ALO technique, FOPI assessor and ALOFOPI assessor. At we can conclude that ALOFOPI assessor powers have been enhanced when compared with the remaining assessors.

\section{Published By:}




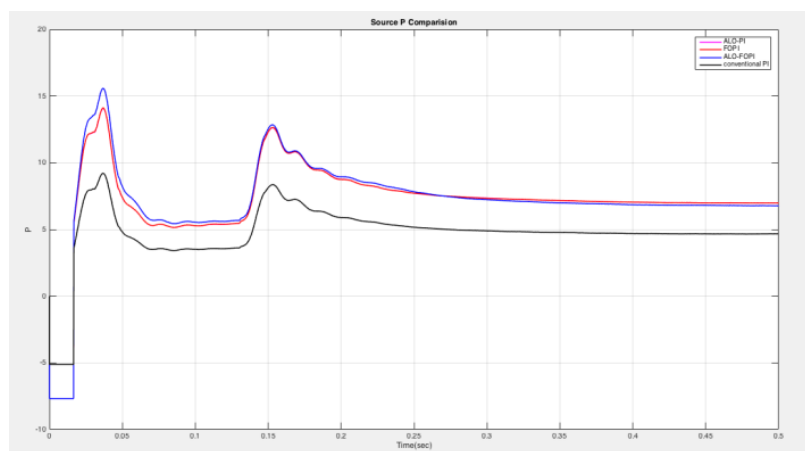

Fig9: Collation of typicalPI,ALO-PI,FOPI,ALOFOPI assessors in generating the active power with a voltage dip from 0.03 to $0.13 \mathrm{msec}$ at source

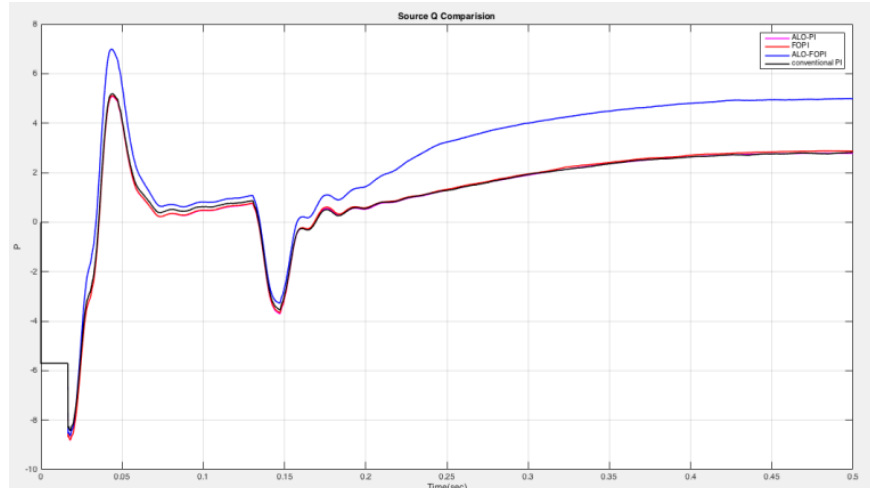

Fig10: Collation of typicalPI,ALO-PI,FOPI,ALOFOPI assessors in generating there- active power with a voltage dip from 0.03 to $0.13 \mathrm{msec}$ at source

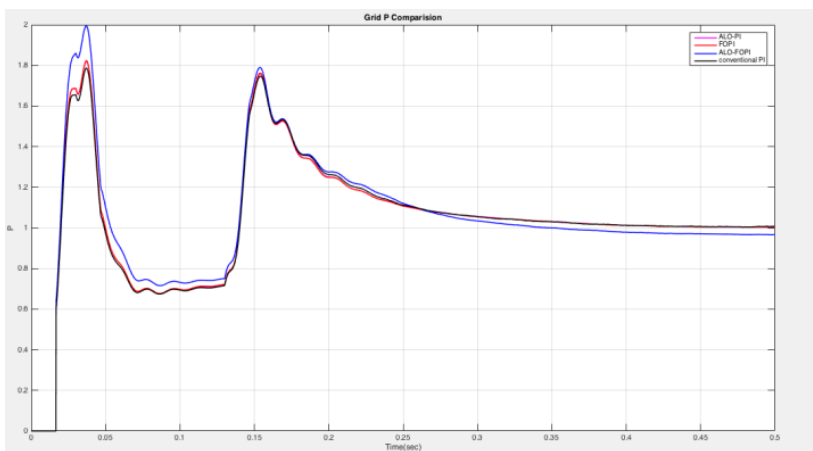

Fig11: Collation of typicalPI,ALO-PI,FOPI,ALOFOPI assessors in generating the active power with a voltage dip from 0.03 to 0.13 msec at grid

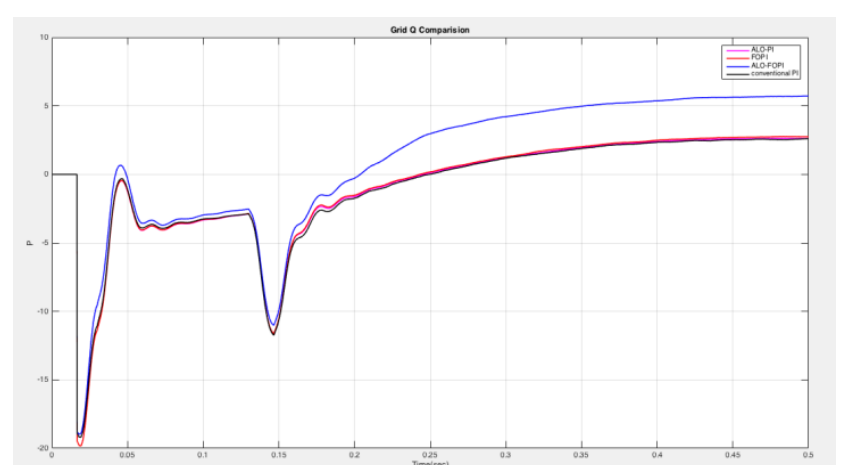

Fig12: Collation of typicalPI,ALO-PI,FOPI,ALOFOPI assessors in generating the active power with a voltage dip from 0.03 to 0.13 msec at grid

\section{CONCLUSION:}

From the simulation results the collated with typical PI controller the power outputs are enhanced with ALOFOPI controllers. It can uses various sizes model for study and monitoringadvanced strategies in future. From the results we can understand that the power output properties can yield much better even with various input velocities of the wind speeds.

\section{REFERENCES:}

1. SeyedaliMirjalili 'The Ant Lion Optimizer' Advances in engineering software.School of Information and Communication Technology, Griffith University, Nathan, Brisbane, QLD 4111, Australia Queensland Institute of Business and Technology, Mt Gravatt, Brisbane, QLD 4122, Australia.

2. T. Unchim and A. OonsivilaiA Study ofWind Speed Characteristic in PI Controller based DFIGWind Turbine World Academy of Science, Engineering and Technology International Journal of Electrical and Computer Vol:5, No:12, 2011

3. An Improved Ant Lion Optimization Algorithm and Its Application in Hydraulic Turbine Governing System Parameter Identification TianTian 1, Changyu Liu 1,*, Qi Guo 2, Yi Yuan 2, Wei Li 2 and Qiurong Yan 3

4. Coordinated control of rotor and grid sides converters in DFIG based wind turbines for providing optimal reactive power support and voltage regulation Mohsen Rahimi Sustainable Energy Technologies and Assessments 20 (2017) 47-57.

5. Hansen,L.H.Helle L., Blaabjerg F., Ritchie E., Munk-Nielsen S.,Binder, H.Sorensen, P. and Bak-Jensen,B.,"conceptual survey of Generators and Power Electronics for Wind Turbines",Rise National Laboratory, Roskilde, Denmark, Riso-R1205(EN),2001

6. Abdelaziz Azza1, Hamid Kherfane2,' Robust Control of Doubly Fed Induction Generator Using Fractional Order Control'International Journal of Power Electronics and Drive System (IJPEDS) Vol. 9, No. 3, September 2018, pp 1072 1080 ISSN: 2088-8694, DOI: 10.11591/ijpeds.v9n3.pp1072-1080 . 